\title{
Communication
}

\section{Micro-Tapered Fiber Few-Mode Interferometers Incorporated by Molecule Self-Assembly Fiber Grating for Temperature Sensing Applications}

\author{
Haimiao Zhou ${ }^{1}$, Lina Suo ${ }^{1}$, Ya-Pei Peng ${ }^{2}$, Fan Yang ${ }^{1}$, Shijie Ren ${ }^{1}$, Nan-Kuang Chen ${ }^{1, *}, X$ inhe Lu ${ }^{3}$, \\ B.M.A. Rahman ${ }^{4}$ (D) and K.T.V. Grattan ${ }^{4}$
}

check for

updates

Citation: Zhou, H.; Suo, L.;

Peng, Y.-P.; Yang, F.; Ren, S.;

Chen, N.-K.; Lu, X.; Rahman, B.;

Grattan, K. Micro-Tapered Fiber

Few-Mode Interferometers

Incorporated by Molecule

Self-Assembly Fiber Grating for

Temperature Sensing Applications.

Photonics 2022, 9, 96. https: / /

doi.org/10.3390/photonics9020096

Received: 14 January 2022

Accepted: 4 February 2022

Published: 7 February 2022

Publisher's Note: MDPI stays neutral with regard to jurisdictional claims in published maps and institutional affiliations.

Copyright: (c) 2022 by the authors. Licensee MDPI, Basel, Switzerland. This article is an open access article distributed under the terms and conditions of the Creative Commons Attribution (CC BY) license (https:/ / creativecommons.org/licenses/by/ $4.0 /)$.
1 School of Physics Sciences and Information Technology, Liaocheng University, Liaocheng 252000, China; 1910110302@stu.lcu.edu.cn (H.Z.); 1920110506@stu.lcu.edu.cn (L.S.); 2010280104@stu.lcu.edu.cn (F.Y.); renshijie@lcu.edu.cn (S.R.)

2 College of Engineering Physics, Shenzhen Technology University, Shenzhen 518000, China; pengyapei@sztu.edu.cn

3 NK Photonics Ltd., Jinan 250119, China; xinhelu@nkphotonics.com

4 School of Mathematics, Computer Science \& Engineering, City, University of London, Northampton Square, London EC1V 0HB, UK; b.m.a.rahman@city.ac.uk (B.M.A.R.); k.t.v.grattan@city.ac.uk (K.T.V.G.)

* Correspondence: nankuang@gmail.com
Abstract: We demonstrate fiber few-mode interferometers based on a self-assembly surface corrugated grating using charged nano-particles. Initially, an abrupt taper (AT) was first created using a micro flame. The AT was then further outwardly stretched to make an elongated uniformed taper until the tapered diameter achieved a micron scale. The high order core modes (HOCMs) were excited at the AT and the optical path difference (OPD) among the modes was enlarged through the uniformed taper to achieve the few-mode interference effects seen. However, to significantly enhance the interference effects with higher extinction ratios (ER) over such a short length of interferometer, an external assisted grating was made using charged nanoparticles to form surface corrugated grating with a period, $\Lambda$, of approximately $14 \mu \mathrm{m}$. This intermediate period of the fiber grating was helpful in scattering and attenuating some unwanted high-order modes to change the optical characteristics of the few-mode interferometer (FMI). This FMI with a self-assembly fiber grating (SAFG) was further used to make fiber temperature sensors, with a maximum resonant wavelength shift of $4.6 \mathrm{~nm}$, over a temperature range from $20-60{ }^{\circ} \mathrm{C}$. The temperature sensitivity achieved was $112.6 \mathrm{pm} /{ }^{\circ} \mathrm{C}$ and the coefficient of determination, $\mathrm{R}^{2}$, was as high as 0.99 , which revealed the high linearity of the results.

Keywords: molecule self-assembly; few-mode interferometer; tapered fiber; fiber grating; charged nano-particles

\section{Introduction}

Over the past few decades, fiber interferometers have been recognized as powerful tools for precisely measuring physical, chemical, or biological parameters in many scientific and industrial applications. Among those fiber interferometers, modal interferometers with high sensitivity have been widely employed for optical imaging and sensing systems [1-3]. However, the long-term environment and temperature stability are always important issues to be addressed and compensated for. Conveniently, fiber gratings, including the Bragg, long-period, phase-shifted, blazed, superstructure, rocking, and chiral gratings [4-10], inscribed in the core or corrugated on the surface of the single-mode fibers (SMF), are another kind of successful interferometer and are more intensively used due to their great precision and high stability. Originally, the fiber Bragg gratings used in such work were made by creating longitudinal standing waves in a Ge-doped SMF over several hours using an Ar ion laser [11-13]. Therefore, high system stability of the experimental set-up needed was stringent for good interference effects to be seen. In addition, various kinds of fabrication 
methods such as dual-beam interference, femtosecond point-by-point writing, chemical etching, phase mask exposure, mechanical pressing, ion beam milling [14-19], and so on have been used in the fabrication of various kinds of fiber gratings since then. To date, the most successful and mature fabrication methods for fiber gratings are based on exploiting the fiber photosensitivity [20,21] where light from a $248 \mathrm{~nm}$ excimer laser or a $244 \mathrm{~nm}$ frequency-doubled laser is used to break the covalent bonds between the germanium and oxygen atoms in the fiber core. The vacancy of the missing electron, forming a color center absorber, induces the periodical changes of the refractive index seen along the Ge-doped fiber core $[22,23]$. However, this method is not suitable for those special fibers, such as no-core fibers and phosphate glass fibers, which have a lack of photosensitivity (such as the no-core fiber or silica micro-/nano-tapered fibers) and are becoming more and more important for key applications in micro- or nano-photonics. Instead, the femtosecond laser writing, chemical etching, or ion beam milling methods available could be used as alternative fabrication methods to create periodical index variations in micro-tapered fibers [24-27]. Nevertheless, the key trade-off issue here is that the mechanical strength is significantly weakened, and this is the effect which is often seen to sacrifice the long-term stability of the devices created in this way.

In contrast to the aforementioned fabrication methods for fiber gratings, the fiber gratings written here in few-mode micro-tapered fibers were based on a new method using molecular self-assembly and this approach is newly proposed and demonstrated in this paper. The combination of the surface-corrugated SAFG and the few-mode tapered fiber $[28,29]$ were also demonstrated to achieve high sensitivity fiber interferometers. The SAFG was formed using the charged nano-particles generated from the smoke derived from the incomplete combustion of the carbon compounds present, with the major materials containing wood tar which is fluxible (i.e., which flows) at temperatures over $82{ }^{\circ} \mathrm{C}$. The wood tar is steamed when the operating temperature rises above $405{ }^{\circ} \mathrm{C}$ and the typical combustion temperature for smoking is usually far beyond this temperature of $400{ }^{\circ} \mathrm{C}$. The micro-tapered silica fiber carries negative charges when its tapered diameter is reduced down to a wavelength scale, namely a few microns. The smoking nano-particles are positively charged and are further attracted to attach themselves to the tapered fiber surface. The distance between the tapered fiber and the heat source is typically around a few centimeters. The individual nano-particles gather together to become larger clusters, distributed almost periodically to achieve the SAFG. The periodicity, $\Lambda$, can be controlled by selecting the appropriate elapsed smoking time, the distance during the smoking process, the tapered diameter, and the nature of the burning materials. An external heating source was also found to be a good option to help control the value of $\Lambda$ achieved. Further, the tapered fiber FMI was made by elongating the value of AT which was formed using a micro-flame to break the waveguide adiabaticity $[30,31]$. Therefore, the HOCMs can be excited through this AT, using a length of approximately $824.6 \mu \mathrm{m}$. To increase the OPD between the HOCMs, the AT was further elongated using a scanning flame until the total tapered length reached $3.21 \mathrm{~mm}$. The spectral oscillations could be seen to be clearly available, due to the HOCMs interference, but the generated ER is typically less than $13 \mathrm{~dB}$. This value of ER can obviously be improved (to become $>20 \mathrm{~dB}$ ) when the SAFG is made on the surface of the tapered fiber, since the SAFG has a higher index than silica, to increase the OPD between HOCMs and to remove some unwanted modes. Therefore, some of the excited modes were significantly scattered and attenuated and the existing numbers of the spectral oscillations were reduced, this being ascribed to the intermediate period of SAFG [32]. Thus, the free spectral range (FSR), as well as the ER for the resonant wavelengths, both increased. Moreover, the FMIs with a SAFG were also used to make fiber temperature sensors. This exploited the maximum resonant wavelength shift of $4.6 \mathrm{~nm}$, over the temperature region studies from $20-60{ }^{\circ} \mathrm{C}$. The temperature sensitivity achieved was $112.6 \mathrm{pm} /{ }^{\circ} \mathrm{C}$ and the coefficient of determination, $\mathrm{R}^{2}$, was as high as 0.99 , indicating the high linearity of the device. This SAFG-based approach, using charged smoking nanoparticles, is a new fabrication method for making compact periodical micro-structures on 
micro- or nano-tapered fibers and is able to give rise to spectral resonances [33] with high ER. In contrast to the chemically etched or laser-ablated surface-corrugated gratings, this method is good in that it does not deteriorate the mechanical strength since the tapered fiber remains smooth and in one piece which prevents the generation of micro-cracks when the micro- or nano-tapered fiber is slightly bent or twisted. Moreover, the SAFG-incorporated FMIs are compact and, thus, can be used as an efficient way to achieve high sensitivity interferometers for the sensing systems needed today.

\section{Experimental Set-Up and Working Principle}

In the fabrication process developed and used, a segment of the SMF was uncoated and then placed and fixed on the fiber taper station, shown in Figure 1a. Broadband white-light (covering the spectral region from 1250-1650 nm, from a superluminescent diode (SLD)) was launched into the fiber, with an Optical Spectrum Analyzer (OSA) used at the other end to measure the spectral response achieved. A micro-hydrogen flame torch was applied to heat the fiber locally, allowing it to be tapered rapidly, to create an abrupt taper, shown in Figure 1b. The L1 and L2 are the total lengths of the abrupt taper and the uniformed taper, respectively. The half difference, (L1-L2) $/ 2=0.395 \mathrm{~mm}$, is the length of one abrupt taper transition. The fiber diameter decreased from $125 \mu \mathrm{m}$ to the tapered diameter, D, of $2.73 \mu \mathrm{m}$, within a very short distance, which, thus, obviously breaks the waveguide adiabaticity to excite the HOCMs. Subsequently, another torch, with a wider flame diameter of $9 \mathrm{~mm}$, was used to heat the abrupt taper point, back and forth, to lead to a longer uniformed tapered length, until an average value of D of $2.73 \mu \mathrm{m}$ was reached, as shown in Figure 1b. The uniformed tapered region in this work was defined as the region with a diameter tolerance $\pm 0.1 \mu \mathrm{m}$ under a $1000 \times$ CCD microscope. From this figure, it can be seen that the shape of the taper transition changed very quickly at the bilateral ends of the uniformed taper. Since the AT can excite HOCMs due to the rapid changes in D, the elongated uniformed taper, $2.42 \mathrm{~mm}$ in length, can help further induce larger values of OPD for good interference due to the higher index difference between the HOCMs. However, the HOCMs are all guided to propagate in the silica tapered fiber, one in which the original fiber cladding and air in the un-tapered fiber turn out to be the new guiding core and new cladding, respectively. In principle, by applying a thin layer of the highindex material over the evanescent field of the tapered fiber, the OPD between the higherand lower-order core modes can, thus, be increased. This occurs because the penetration depth of the evanescent fields for the HOCMs can be extended further away from the lower-order modes. Interestingly, when the high-index thin overlay on the tapered fiber is periodically distributed, the spectral responses for this interferometer will be different. First, the high-index overlay can reduce the number of resonances by attenuating some unwanted HOCMs, which then increases the value of the FSR. Second, the ER used for interference effects to be seen can be significantly improved using the most suitable ranges of overlay thickness. To achieve the periodically distributed high-index thin overlay on the tapered fibers, a new fabrication method has been proposed in this work. A large amount of the positively charged nano-particles are generated from combustion using the fumes, the so-called 'incenses' which are a major ingredient of the wood tar. At temperatures $>82{ }^{\circ} \mathrm{C}$, this material 'flows' and becomes steamed when the temperature is $>405^{\circ} \mathrm{C}$. Under typical conditions, the combustion temperature when using such incense is usually far beyond $400{ }^{\circ} \mathrm{C}$. Thus, the wood tar molecules were steamed from the beginning and then liquefied when arriving at the tapered fiber surface, at a position $1.5 \mathrm{~cm}$ above the combustion source, as shown schematically in Figure 2a. For the steamed molecules, the molecules are ionized to be in a plasma phase. Since the electrons are much lighter than the particles in the air, the electrons rapidly escape and disappear. The remaining particles are heavier and, thus, positively charged. They rise with the hot air flow, to reach the tapered fiber surface. Owing to the static charges, the charged liquefied wood tar molecules strongly repel each other to become periodically distributed, as shown in Figure 2b. More and more charged molecules coming from the rising smoke arrive to join the existing molecules, which allow 
the clustered molecules to keep growing in size, as well as the value of $\Lambda$ of the distributed molecules increasing before saturation occurs. By selecting the most suitable parameters for making the SAFG, the quasi-periodic gratings created can be used to serve as fiber gratings. In Figure $2 c$, it is indicated that a value of $\Lambda$ corresponding to the short-, intermediate-, and long-period gratings are roughly categorized into the following groups: $<1 \mu \mathrm{m}$, between 1-100 $\mu \mathrm{m}$, and $>100 \mu \mathrm{m}$, respectively. The short-period grating, also called a Bragg grating, has a grating vector in an inverse way with respect to the lightwave propagation direction, to reflect backward the phase-matching wavelength, namely the Bragg wavelengths. In contrast, the long-period grating can couple the phase-matching wavelengths to forward propagate in the cladding. When the value of $\Lambda$ is located somewhere between the short and long period, this intermediate-period grating can scatter the light used radially [32]. Therefore, in contrast to the conventional fiber long-period gratings or Bragg gratings which are most familiar, this SAFG has a value of $\Lambda$ at $14 \mu \mathrm{m}$, which would lead to the effect of scattering and attenuating certain HOCMs. This intermediate period of a surfacecorrugated fiber grating [32] incorporated into a compact few-mode fiber interferometer can be used to efficiently increase the ER over a short length $(<3.2 \mathrm{~mm})$ footprint, ascribed to the higher index of the wood tar molecules which are in a periodical distribution on the tapered fibers.

(a)

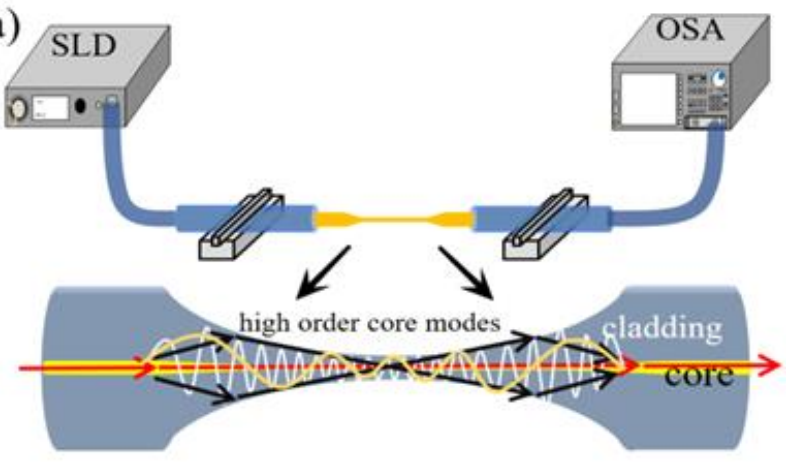

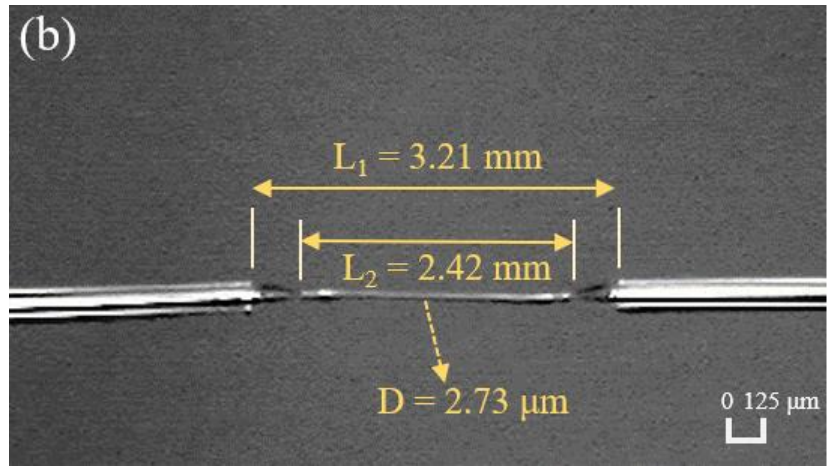

Figure 1. (a) Experimental set-up and (b) microphotograph of the FMI under a 50× CCD microscope.

(a)

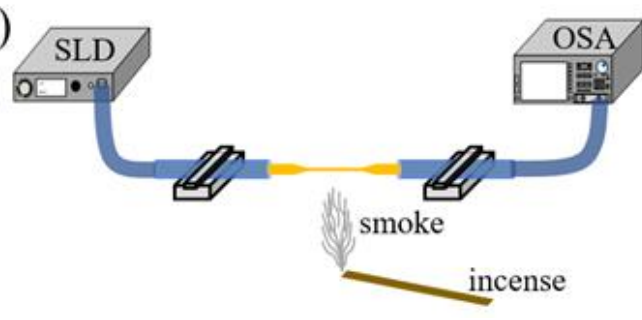

(b)

clustered nano-particles

(c)

$$
\text { short period }(<1 \mu \mathrm{m})
$$
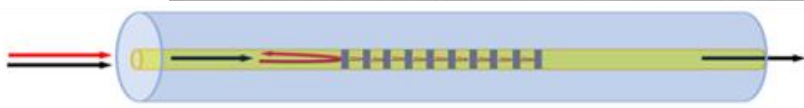

$\Lambda=16.23 \mu \mathrm{m}$
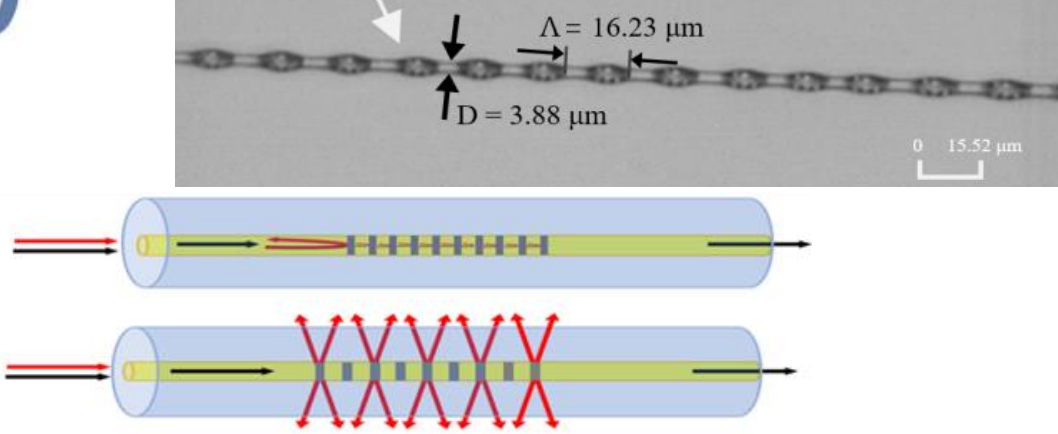

intermediate period $(1 \mu \mathrm{m}<\Lambda<100 \mu \mathrm{m})$

long period $(>100 \mu \mathrm{m})$

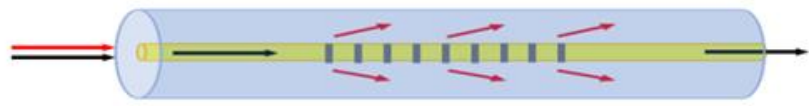

Figure 2. (a) Schematic diagram of the SAFG-incorporated FMI; (b) Microphotograph of the SAFG under a $1000 \times$ CCD microscope; (c) Schematic description of grating vectors for short-, intermediate-, and long-period grating. 


\section{Measurements and Discussion}

To investigate the optical characteristics of the tapered fiber FMI incorporated in the SAFG, a test sample was prepared with $\mathrm{D}=2.73 \mu \mathrm{m}$. The white-light from SLDs was launched into the interferometer and the spectral response achieved was recorded by using the OSA. Figure 3a shows a microphotograph of sample A (with $\mathrm{D}=2.73 \mu \mathrm{m}$ ), taken using a $1000 \times$ CCD microscope. When the SAFG was made of charged wood tar molecules from the burning incense, the value of $\Lambda$ was $14.01 \mu \mathrm{m}$, as shown in Figure $3 \mathrm{~b}$. The original spectral response of the FMI without the SAFG is shown as the red line in Figure 3c. It was found that the value of ER was typically less than $10 \mathrm{~dB}$. When the tapered fiber was incorporated in the SAFG, the spectral response changed, as shown by the blue line in Figure 3c. As mentioned above, the insertion loss, as well as the FSR, increased by a small amount due to the higher index of the wood tar, compared with that of the silica fiber. Obviously, the ER can be substantially enhanced to become higher than $20 \mathrm{~dB}$. This SAFG is quite robust and can only be partially removed by applying an alcohol spray several times in order to investigate the repeatable use of the few-mode interferometer after making and removing the SAFG. In Figure $3 d$, the red line shows the spectrum of the few-mode interferometer without SAFG on the tapered fiber. The green line shows the spectrum of the interferometer after the first SAFG was made and then removed by alcohol. The green line almost overlaps with the red line at all the wavelengths. By continuously repeating the process again, the blue line shows the spectrum of the interferometer after the second SAFG was made and then removed. It is found that the blue line cannot overlap well with the red line due to some contaminant. Thus, the interferometer should be further cleaned using some strong chemicals like sulfuric acid to improve the repeatability of the interferometers for making SAFG. Figure 3e shows the grating formation evolution with the elapsed time, $\mathrm{T}$, at values where $\mathrm{T}=0.5, \mathrm{~T}=2$, and $\mathrm{T}=4 \mathrm{~s}$. The SAFG was initially formed to change the periodical oscillation of the FMI at T $=0.5 \mathrm{~s}$. Subsequently, the FSR, the insertion loss, and the ER change with time to create a better result at $\mathrm{T}=2 \mathrm{~s}$, after which the spectral responses of the SAFG would be seen to have been downgraded when too many smoking particles join to deform the periodic structure. Thus, in summary, this SAFG can be recognized as a new fabrication method for making fiber gratings, especially suited for use on special fibers, like no-core fibers and phosphate glass fibers, which show a lack of photosensitivity and the required good mechanical strength. The value of $\Lambda$ needed can be determined by selecting the most appropriate elapsed smoking time, the distance between the fiber and combustion source, and the burning materials chosen. In this work, the effect was demonstrated where the wood tar was steamed and positively charged at the beginning of the experiment. Following that, when the heating temperature was less than $405^{\circ} \mathrm{C}$, the molecules were seen to be clustered into periodical groups on the tapered fibers, forming gratings automatically. When the temperature was below $82{ }^{\circ} \mathrm{C}$, the SAFG was stabilized and the value of $\Lambda$ was then determined. The ER can be substantially enhanced (to be above $20 \mathrm{~dB}$ ) for the tapered fiber FMI, only with a device length of $3.2 \mathrm{~mm}$.
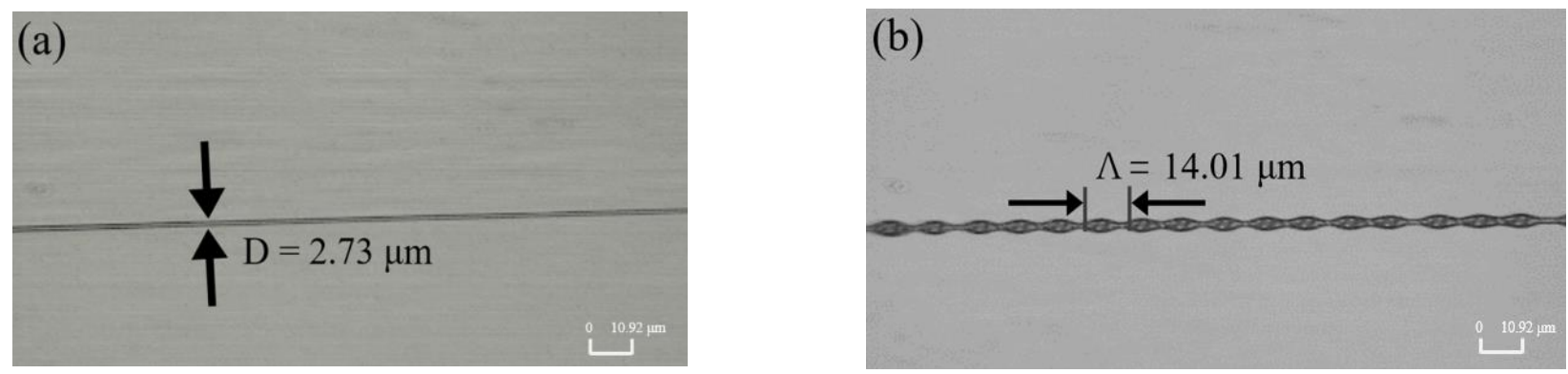

Figure 3. Cont. 

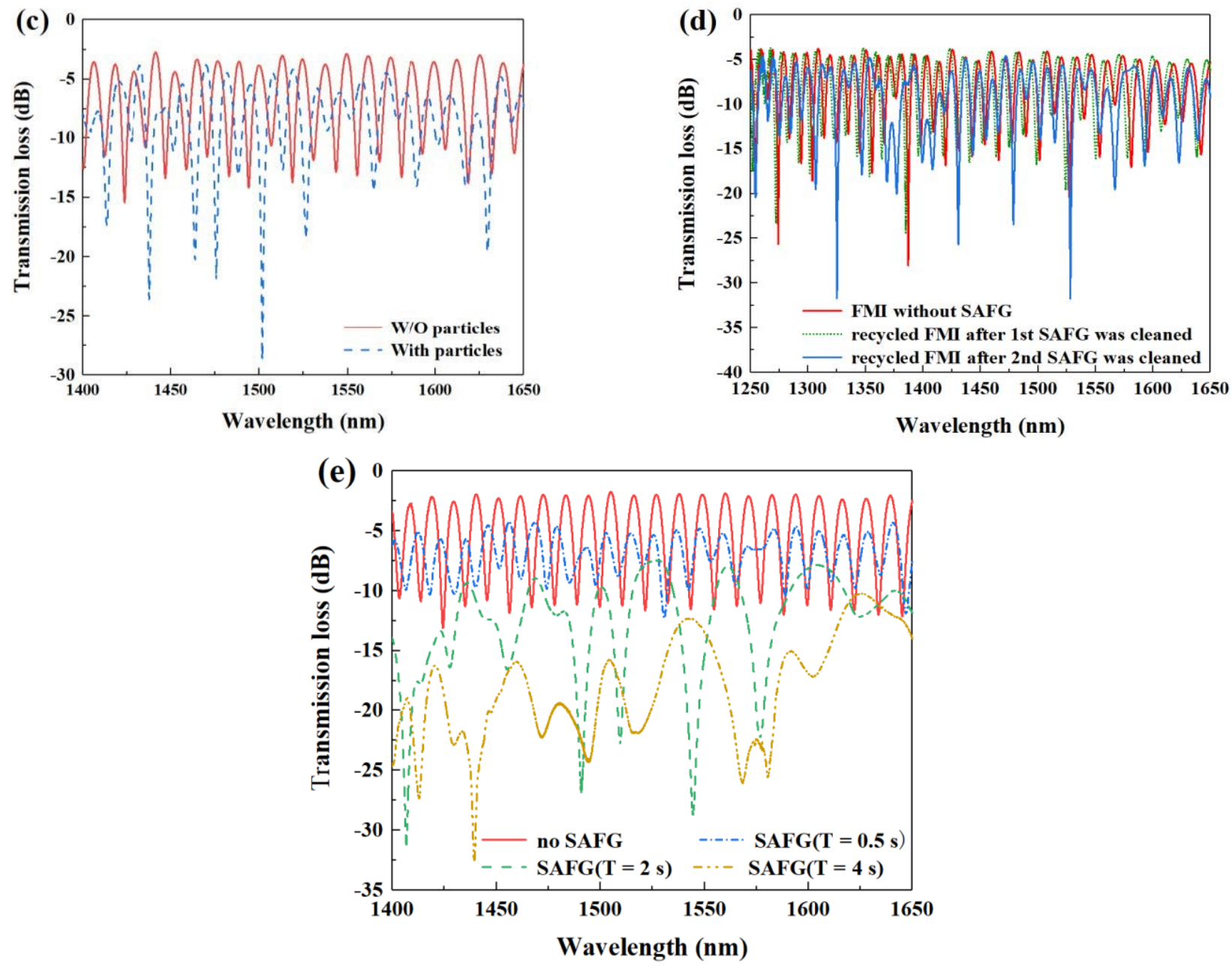

Figure 3. Microphotographs of the (a) tapered fiber and (b) SAFG-incorporated FMI under the $1000 \times$ CCD microscope. Spectral responses of the (c) FMI versus SAFG-incorporated FMI and (d) the repeatability for the SAFG-incorporated FMI using alcohol spray. (e) Spectral evolutions during the SAFG formation at different elapsed time, $T$.

To investigate the potential applications of the grating created in temperature sensing, sample B with a $\mathrm{D}=3.5 \mu \mathrm{m}$, was used. Sample B was placed inside a temperature controlled cavity, having a temperature resolution of $0.1^{\circ} \mathrm{C}$. The sample B used had a SAFG period of $\sim 13 \mu \mathrm{m}$ and the heating temperature was controlled over a range from $20^{\circ} \mathrm{C}$ to $60^{\circ} \mathrm{C}$ (with an increment of $5^{\circ} \mathrm{C}$ ) in the experiment carried out. As mentioned above, the carbon compounds present with the major materials containing wood tar and the SAFG can be stabilized at the temperature below $82{ }^{\circ} \mathrm{C}$. However, for temperature sensing measurements, the heating time in the oven is approximately $20 \mathrm{~min}$ for stabilization and accuracy. However, it was observed that the spectral responses start to become unstable when the heating temperature is above $65^{\circ} \mathrm{C}$ for over $20 \mathrm{~min}$. In fact, the heating temperature should always be below $82{ }^{\circ} \mathrm{C}$ to prevent serious SAFG deformation. However, when the heating temperature is increased to $65^{\circ} \mathrm{C}$, some ingredients of the carbon compounds leak out to lead to index instability or slight structure deformation that cannot be observed by naked eyes. Thus, the temperature sensing is carried out between 20 and $60{ }^{\circ} \mathrm{C}$ in this work. Some pure chemicals with higher softening temperatures could be useful to improve the temperature sensing range in future works. In addition, the grating pitch can be determined by changing the heating temperature of the smoke or the distance between the tapered fiber and the smoking source to change the viscosity of the clustered particles on tapered fibers. In addition, the grating pitch can also be affected by the adhesion between the tapered fiber 
and the cluster particles and the tapered fiber diameter. In our experiments, it was found that a smaller tapered diameter can normally lead to a somehow shorter grating pitch. However, this is a complicated process due to several issues including the temperature and ingredients of the smoking particles, the distance between the tapered fiber and the smoking source, the tapered diameter, and the adhesion between the tapered fiber and the clustered particles. Obviously, the temperature and the viscosity dominate the issue of determining the grating pitch. Figure $4 \mathrm{a}$ shows the spectral responses achieved from the investigation, under different heating temperatures being used. The calibration for the device can be seen in Figure 4. The resonant wavelength blue-shift seen with increasing temperature was as shown in Figure 4 b. From this figure, the temperature sensitivity, $S$, can be deduced to be $112.6 \mathrm{pm} /{ }^{\circ} \mathrm{C}$, an important result, and which is at least twice that seen with other kinds of temperature sensors using special fibers or interferometers [34-36]. Further confidence is given in the result in that the coefficient of determination, $\mathrm{R}^{2}$ is 0.99 , which reveals the high linearity of the wavelength shift, $\Delta \lambda$, achieved as a function of the temperature variations in the test oven. The relationship between the value of $\Delta \lambda$ and the temperature can be seen more clearly in Figure 4c. In short, the merits of this temperature sensor include the following points: (1) a new fabrication technique to make fiber gratings on micro-tapered fiber based on the self-assembly particles in quasi-periodic distributions, (2) a temperature sensitivity of up to $112.6 \mathrm{pm} /{ }^{\circ} \mathrm{C}$ which is higher than those SMS (single-mode/multi-mode/single mode)-based temperature sensors [37], and (3) a compact fiber sensor with a footprint of less than $4 \mathrm{~mm}$ (total length of the interferometer is $3.21 \mathrm{~mm})$.
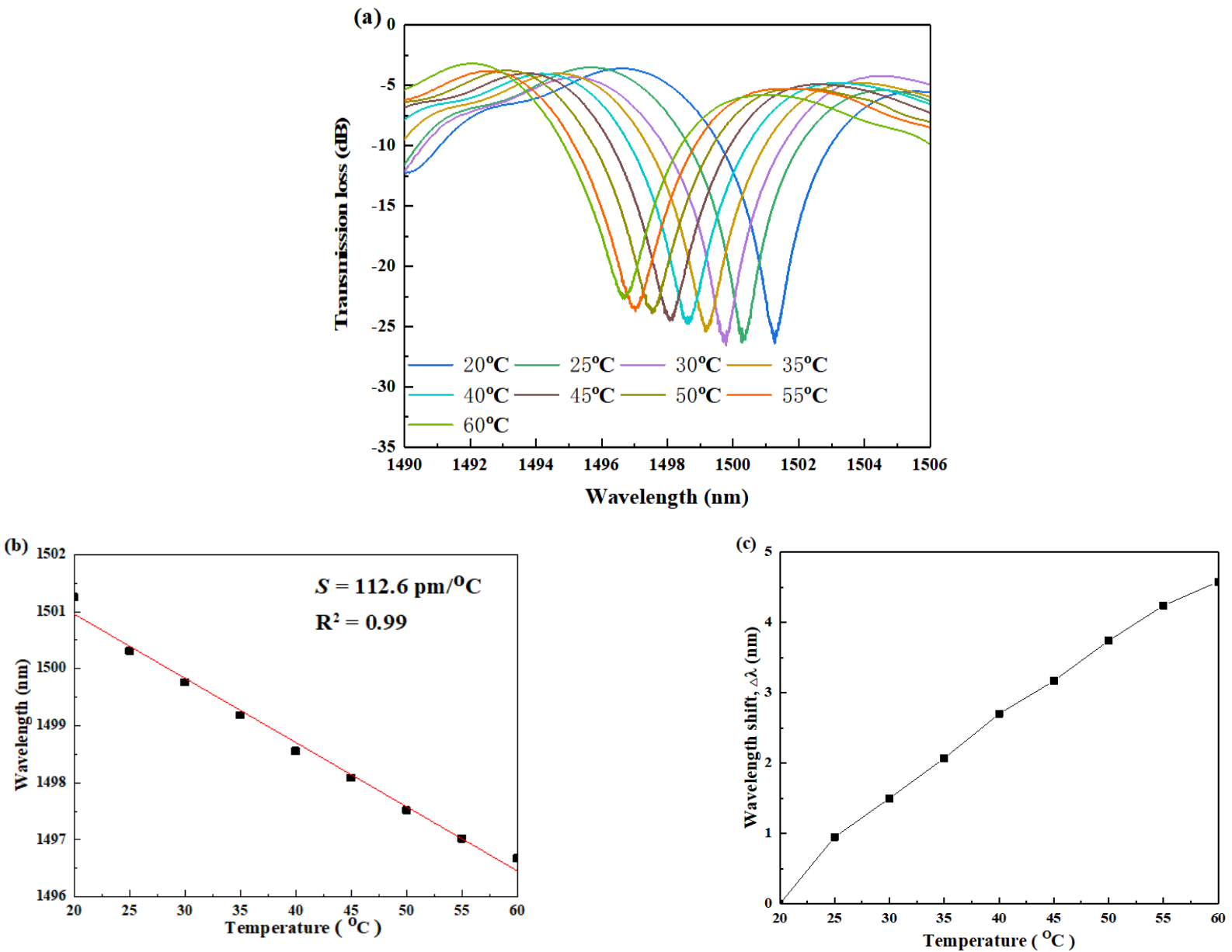

Figure 4. (a) Spectral responses of the FMI with SAFG under different heating temperatures. (b) Resonant wavelengths versus different heating temperatures. (c) Wavelength shift with the oven heating temperatures. 


\section{Conclusions}

In this work, a new fabrication method for making effective fiber gratings on microtapered fibers has been proposed and the results of an investigation discussed. This SAFG has been incorporated into an FMI to increase the ER (to be above $20 \mathrm{~dB}$ ), within a short device length of $<3.2 \mathrm{~mm}$. The FMI was made by elongating an AT on a standard SMF to excite first the HOCMs to achieve the desired interference effects. The incorporation of the SAFG can efficiently enhance the ER (to be above $20 \mathrm{~dB}$ ) due to the higher index of the wood tar materials from the burning incense that is used. The wood tar molecules were positively charged when they were steamed through the combustion process used. The positively charged molecules adhered to the tapered fiber and then clustered into periodical groups, to form fiber gratings, with an intermediate period of $14.01 \mu \mathrm{m}$ to remove the unwanted HOCMs. The grating created can be stabilized when the heating temperature used is less than the softening point of the materials. The grating can, if needed, be removed using alcohol spray (applying this several times for full removal). However, a SAFG with stronger mechanical characteristics can be achieved by selecting materials with strong chemical bonds, such as the use of an epoxy resin. This SAFG-assisted FMI shows a very good result, with a high ER within a device $3.2 \mathrm{~mm}$ in length. For temperature sensing applications, the maximum resonant wavelength shift seen was $4.6 \mathrm{~nm}$ (over the temperature range studied, from $20^{\circ} \mathrm{C}$ to $60^{\circ} \mathrm{C}$ ). The temperature sensitivity achieved was $112.6 \mathrm{pm} /{ }^{\circ} \mathrm{C}$ and this result is higher than that achieved for many temperature sensors using special fibers or interferometers. Further, the coefficient of determination, $\mathrm{R}^{2}$, is as high as 0.99 indicating the high linearity of the result. This new fabrication method for fiber gratings is advantageous as it enables gratings to be made on those fibers which show a lack of photosensitivity. Compared with the chemical etched or laser-ablated surface-corrugated gratings, this method can also help make gratings with better mechanical strength in micro-/nano-tapered fibers. This is because the tapered fiber remains smooth and in one piece to prevent the generation of micro-cracks when the micro- or nano-tapered fiber is slightly bent or twisted. Moreover, the grating pitch can be readily determined by selecting the appropriate smoking time used, the distance between the fiber and the combustion source, the tapered fiber diameter, and the burning materials used, to allow different optical characteristics to be achieved for use in many applications today.

Author Contributions: Conceptualization, N.-K.C. and H.Z.; methodology, H.Z. and S.R.; validation, Y.-P.P.; formal analysis, H.Z.; investigation, L.S., X.L. and H.Z.; resources, N.-K.C.; data curation, S.R.; writing-original draft preparation, H.Z.; writing—review and editing, B.M.A.R., K.T.V.G. and F.Y.; supervision, N.-K.C.; project administration, N.-K.C.; funding acquisition, N.-K.C. All authors have read and agreed to the published version of the manuscript.

Funding: This research was funded by the National Natural Science Foundation of China, grant number 61875247. It was also supported by Liaocheng University under Grants 31805180101 and 319190301.

Institutional Review Board Statement: Not applicable.

Informed Consent Statement: Not applicable.

Data Availability Statement: The data that support the findings of this study are available upon request from the corresponding author. The data are not publicly available due to privacy or ethical restrictions.

Acknowledgments: Partial financial supports from the National Natural Science Foundation of China and Liaocheng University, Shandong, China, are highly appreciated.

Conflicts of Interest: The authors declare no conflict of interest.

\section{References}

1. Dong, S.D.; Dong, B.; Yu, C.Y.; Guo, Y.X. High sensitivity optical fiber curvature sensor based on cascaded fiber interferometer. J. Lightwave Technol. 2018, 36, 1125-1130. [CrossRef] 
2. Avila-Garcia, M.S.; Bianchetti, M.; Corre, R.L.; Guevel, A.; Mata-Chavez, R.L.; Sierra-Hernandez, J.M.; Jauregui-Vazquez, D.; Reyes-Ayona, J.R.; Wstudillo-Ayala, J.M.; Rojas-Laguna, R. High sensitivity strain sensors based on single-mode-fiber core-offset Mach-Zehnder interferometers. Opt. Lasers Eng. 2018, 107, 202-206. [CrossRef]

3. Chen, E.Q.; Dong, B.; Li, Y.; Wang, X.L.; Zhao, Y.D.; Xu, W.; Zhao, W.; Wang, Y.S. Cascaded few-mode fiber down-taper modal interferometers and their application in curvature sensing. Opt. Commun. 2020, 475, 126274. [CrossRef]

4. Erdogan, T.; Mizrahi, V. Characterization of UV-induced birefringence in photosensitive Ge-doped silica optical fibers. J. Opt. Soc. Am. B 1994, 11, 2100-2105. [CrossRef]

5. Hill, K.O.; Meltz, G. Fiber Bragg grating technology fundamentals and overview. J. Lightwave Technol. 1997, 15, 1263-1276. Available online: https:/ / ieeexplore.ieee.org/document/618320 (accessed on 11 January 2022). [CrossRef]

6. Feng, J.B.; Zhou, Z.P. Polarization beam splitter using a binary blazed grating coupler. Opt. Lett. 2007, 32, 1662-1664. [CrossRef] [PubMed]

7. Cameron, R.P.; Yao, A.M.; Barnet, S.M. Diffraction gratings for chiral molecules and their applications. J. Phys. Chem. A 2014, 118, 3472-3478. [CrossRef] [PubMed]

8. Sun, C.T.; Wang, R.; Jin, X.R.; Wang, Z.M.; Liu, W.L.; Zhang, S.; Zhang, Y.W.; Lin, J.Y.; Li, Y.; Geng, T.; et al. A new phase-shifted long-period fiber grating for simultaneous measurement of torsion and temperature. Chin. Opt. Lett. 2020, 2, 39-43. [CrossRef]

9. Liu, K.; Neo, D.W.K.; Bi, G.J.; Liu, S.B.; Xu, P.; Wang, H.; Nichlas, T.Y.J.; Low, D.W.W.; Zhou, G.Y. Diamond shaping of blazed gratings on freeform surfaces. Precis. Eng. 2021, 72, 899-911. [CrossRef]

10. Xu, F.; Kou, J.L.; Luo, W.; Lu, Y.Q. Surface-Corrugated Microfiber Bragg Grating. In Proceedings of the 2012 Society of PhotoOptical Instrumentation Engineers (SPIE): (SPIE, 2012), Optoelectronic Devices and Integration IV, Beijing, China, 27 November 2012; Volume 8555, p. 23. Available online: https:/ /www.spie.org/Publications/Proceedings/Paper/10.1117/12.999530 (accessed on 6 December 2021).

11. Erdogan, T. Fiber grating spectra. J. Lightwave Technol. 1997, 15, 1277-1294. Available online: https://ieeexplore.ieee.org/ document/618322 (accessed on 13 January 2022). [CrossRef]

12. Yamashita, S.; Baba, T.; Kashiwagi, K. Frequency-Shifted Multiwavelength FBG Laser Sensor. In Proceedings of the 2002 15th Optical Fiber Sensors Conference Technical Digest (OFS), Portland, OR, USA, 7 August 2002; pp. 285-288. Available online: https:/ /ieeexplore.ieee.org/document/1000558 (accessed on 6 January 2022).

13. Cuadrado-Laborde, C.; Díez, A.; Cruz, J.L.; Andrés, M.V. Doubly active Q switching and mode locking of an all-fiber lase. Opt. Lett. 2009, 34, 2709-2711. [CrossRef]

14. Li, X.; Bohn, P.W. Metal-assisted chemical etching in $\mathrm{HF} / \mathrm{H}_{2} \mathrm{O}_{2}$ produces porous silicon. Appl. Phys. Lett. 2000, 77, $2572-2574$. [CrossRef]

15. Iadicicco, A.; Cusano, A.; Cutolo, A.; Bernini, R.; Giordano, M. Thinned fiber Bragg gratings as high sensitivity refractive index sensor. IEEE Photon. Technol. Lett. 2004, 16, 1149-1151. Available online: https://ieeexplore.ieee.org/document/1281900 (accessed on 17 December 2021). [CrossRef]

16. Chartier, C.; Bastide, S.; Lévy-Clément, C. Metal-assisted chemical etching of silicon in HF-H2O2. Electrochim. Acta 2008, 53, 5509-5516. [CrossRef]

17. Xu, S.; Zheng, Y.; Liu, Y.; Liu, W. Intensity clamping during dual-beam interference. Laser Phys. 2010, 20, 1968-1972. Available online: https:/ /link.springer.com/article/10.1134/S1054660X10210139 (accessed on 17 December 2021). [CrossRef]

18. Fang, X.; Liao, C.R.; Wang, D.N. Femtosecond laser fabricated fiber Bragg grating in microfiber for refractive index sensing. Opt. Lett. 2010, 35, 1007-1009. [CrossRef]

19. Ding, M.; Zervas, M.N.; Brambilla, G. A compact broadband microfiber Bragg grating. Opt. Express 2011, 19, 15621-15626. [CrossRef]

20. Xie, X.D.; Li, J.; Sun, L.P.; Shen, X. A High-sensitivity current censor utilizing CrNi wire and microfiber coils. Sensors 2014, 14, 8423-8429. [CrossRef]

21. Liu, S.; He, G.H.; Zheng, Z.; Ding, L.Y.; Zhou, A.; Gou, H.Y.; Zhou, C.M.; Jiang, D. Importance of internal tensile stress in forming low-loss fiber draw-tower gratings. J. Lightwave Technol. 2019, 38, 1900-1904. Available online: https://ieeexplore.ieee.org/ document/8939368 (accessed on 18 December 2021). [CrossRef]

22. Jiang, W.B.; Friberg, S.R.; Iwamura, H.; Yamamoto, Y. Collision-induced pulse shortening in a mode-locked linear-cavity NaCl color-center laser with an InGaAs/InP multiple-quantum-well saturable absorber. Opt. Lett. 1991, 16, 1165-1167. [CrossRef]

23. Oliveira, R.A.; Cook, K.; Canning, J.; Pohl, A.A. Controlling the Properties of Fiber Bragg Gratings by using Acoustic Waves. In Proceedings of the 2010 Bragg Gratings, Photosensitivity, and Poling in Glass Waveguides (BGPP), Karlsruhe, Germany, 21-24 June 2010; p. JThA48. [CrossRef]

24. Zibaii, M.I.; Kazemi, A.; Latififi, H.; Azar, M.K.; Hosseini, S.M.; Ghezelaiagh, M.H. Measuring bacterial growth by refractive index tapered fifiber optic biosensor. J. Photochem. Photobiol. B 2010, 101, 313-320. [CrossRef]

25. Zhu, S.; Pang, F.; Huang, S.; Zou, F.; Dong, Y.; Wang, T. High sensitivity refractive index sensor based on adiabatic tapered optical fifiber deposited with nanofifilm by ALD. Opt. Express 2015, 23, 13880-13888. [CrossRef] [PubMed]

26. Zhao, Y.; Li, X.G.; Cai, L. A highly sensitive Mach-Zehnder interferometric refractive index sensor based on core-offset single mode fiber. Sens. Actuators A 2015, 223, 119-124. [CrossRef]

27. Wang, P.F.; Brambilla, G.; Ding, M.; Semenova, Y.; Wu, Q.; Farrell, G. High-sensitivity, evanescent field refractometric sensor based on a tapered, multimode fifiber interference. Opt. Lett. 2011, 36, 2233-2235. [CrossRef] [PubMed] 
28. Izrailev, F.M.; Makarov, N.M.; Rendon, M. Manifestation of the roughness-square-gradient scattering in surface-corrugated waveguides. Phys. Rev. B 2005, 73, 155421. [CrossRef]

29. Zhang, Q.; Li, J.Y.; Yuan, Y.L.; Gao, L.B. Micro double tapered optical fiber sensors based on the evanescent field-effect and surface modification. Optik 2014, 124, 4614-4617. [CrossRef]

30. Graefe, E.M.; Mailybaev, A.A.; Moiseyev, N. Breakdown of adiabatic transfer of light in waveguides in the presence of absorption. Phys. Rev. A 2013, 88, 9293-9303. [CrossRef]

31. Kwon, J.Y.; Bakti, A.N.; Cho, C.; Kuhlmann, K. Adiabatic performance evaluation system for waveguide transmission lines. IEEE Trans. Instrum. Meas. 2020, 70, 1-9. [CrossRef]

32. Kopp, V.I.; Churikov, V.M.; Jonathan, S.; Norman, C.; Daniel, N.; Azriel, Z.G. Chiral fiber gratings. Science 2004, $305,74-75$. [CrossRef]

33. Moreno, F.; Albella, P.; Nieto-Vesperinas, M. Analysis of the spectral behavior of localized plasmon resonances in the near- and far-field regimes. Langmuir 2013, 29, 6715-6721. [CrossRef]

34. Harris, J.; Lu, P.; Larocque, H.; Xu, Y.P.; Chen, L.; Bao, X.Y. Highly sensitive in-fiber interferometric refractometer with temperature and axial strain compensation. Opt. Express 2013, 21, 9996-10009. [CrossRef] [PubMed]

35. Zhao, Y.; Xia, F.; Hu, H.F.; Du, C. A Ring-Core Optical Fiber Sensor with Asymmetric LPG for Highly Sensitive Temperature Measurement. IEEE Trans. Instrum. Meas. 2017, 66, 3378-3386. [CrossRef]

36. Bae, H.; Yun, D.; Liu, H.; Olson, D.A.; Yu, M. Hybrid Miniature Fabry-Perot Sensor with Dual Optical Cavities for Simultaneous Pressure and Temperature Measurements. J. Lightwave Technol. 2014, 32, 1585-1593. [CrossRef]

37. Wu, Q.; Qu, Y.W.; Liu, J.; Yuan, J.H.; Wang, S.P.; Wu, T.; He, X.D.; Liu, B.; Liu, D.J.; Ma, Y.Q.; et al. Singlemode-MultimodeSinglemode Fiber Structures for Sensing Applications-A Review. IEEE Sens. J. 2021, 21, 12734-12751. [CrossRef] 